\title{
Editorial: State-dependent brain computation
}

\author{
Petra Ritter ${ }^{1,2,3,4 *}$, Viktor K. Jirsa ${ }^{5}$, Anthony R. Mclntosh ${ }^{6}$ and Michael Breakspear ${ }^{7,8}$ \\ ${ }^{1}$ Minerva Research Group Brain Modes, Max Planck Institute for Human Cognitive and Brain Sciences, Leipzig, Germany, \\ ${ }^{2}$ Deparment of Neurology, Charité - University Medicine, Berlin, Germany, ${ }^{3}$ Bernstein Focus State Dependencies of Learning \\ and Bernstein Center for Computational Neuroscience, Berlin, Germany, ${ }^{4}$ Berlin School of Mind and Brain and Mind and \\ Brain Institute, Humboldt University, Berlin, Germany, ${ }^{5}$ Institut de Neurosciences des Systèmes UMR INSERM 1106, \\ Aix-Marseille Université Faculté de Médecine, Marseille, France, ${ }^{6}$ Rotman Research Institute of Baycrest Centre, University of \\ Toronto, Toronto, ON, Canada, ${ }^{7}$ Systems Neuroscience Group, QIMR Berghofer, Brisbane, QLD, Australia, ${ }^{8}$ The Royal \\ Brisbane and Woman's Hospital, Brisbane, QLD, Australia
}

Keywords: state-dependence, computational neuroscience, brain scales, computational modeling, empirical research

The brain is a self-organizing system, which has evolved such that neuronal responses and related behavior are continuously adapted with respect to the external and internal context. This powerful capability is achieved through the modulation of neuronal interactions depending on the history of previously processed information. In particular, the brain updates its connections as it learns successful versus unsuccessful strategies. The resulting connectivity changes, together with stochastic processes (i.e., noise) influence ongoing neuronal dynamics. The role of such statedependent fluctuations may be one of the fundamental computational properties of the brain, being pervasively present in human behavior and leaving a distinctive fingerprint in neuroscience data. This development is captured by the present Frontiers Research Topic, "State-Dependent Brain Computation."

The Research Topic provides an account of prevailing concepts and theories plus recent advances on the role of ongoing brain dynamics-reflecting experiences, global brain states, context and noise-for task-related information processing. Works from the conceptual, experimental and computational-modeling domains are show-cased, focusing on the following two issues: (1) Generative mechanisms of ongoing neuronal dynamics, and (2) Principles of interaction between ongoing dynamics and perceptual or motor processes.

A wide range of spatial and temporal scales encountered in brain dynamics are covered, i.e.,

\section{OPEN ACCESS}

Edited and reviewed by:

Si Wu,

Beijing Normal University, China

*Correspondence: Petra Ritter, petra.ritter@charite.de

Received: 30 May 2015 Accepted: 10 June 2015 Published: 23 June 2015

Citation:

Ritter $P$, Jirsa VK, McIntosh AR and Breakspear M (2015) Editorial: State-dependent brain computation. Front. Comput. Neurosci. 9:77. doi: 10.3389/fncom.2015.00077 from microscopic molecular to macroscopic population dynamics and from fast processes evolving within milliseconds to slow ones taking hours or longer (Table 1). An overview article about statedepended learning exemplifies the need for integration of different scales of processing (Ritter et al., 2015). The role of ongoing alpha oscillations at the microscopic and macroscopic scale for learning is illuminated in Sigala et al. (2014). In this study, the authors present empirical data along with computational models that seek to unveil the underlying principles how oscillations interact with synaptic plasticity. EEG dynamics are also explored in Betzel et al. (2012) where the authors report fast synchronization dynamics - in the range of tens to hundreds of milliseconds-iterating amongst a small set of core networks in the resting brain. The authors suggest that these dynamics may be the neural correlate of resting state BOLD fluctuations. The ability of stochastic dynamic causal modeling (DCM) for fMRI-a neural field formulation of cortical activity - is probed in Daunizeau et al. (2012) where EEG spectral changes are predicted from BOLD signal Fast and high spatial frequency modes as represented in EEG are enslaved by slow and slow spatial frequency modes predominant in fMRI signals. Using an Ising spin model (Deco et al., 2012) demonstrate that the dynamic repertoire of the brain, i.e., different spatio-temporal patterns of functional connectivity, emerges naturally from the neuroanatomical connectivity. It is hypothesized that the scale-free neuroanatomical architecture maximizes the dynamic repertoire and its accessibility in 
TABLE 1 | Different facets of state-dependent brain computation are illuminated in the present Research Topic.

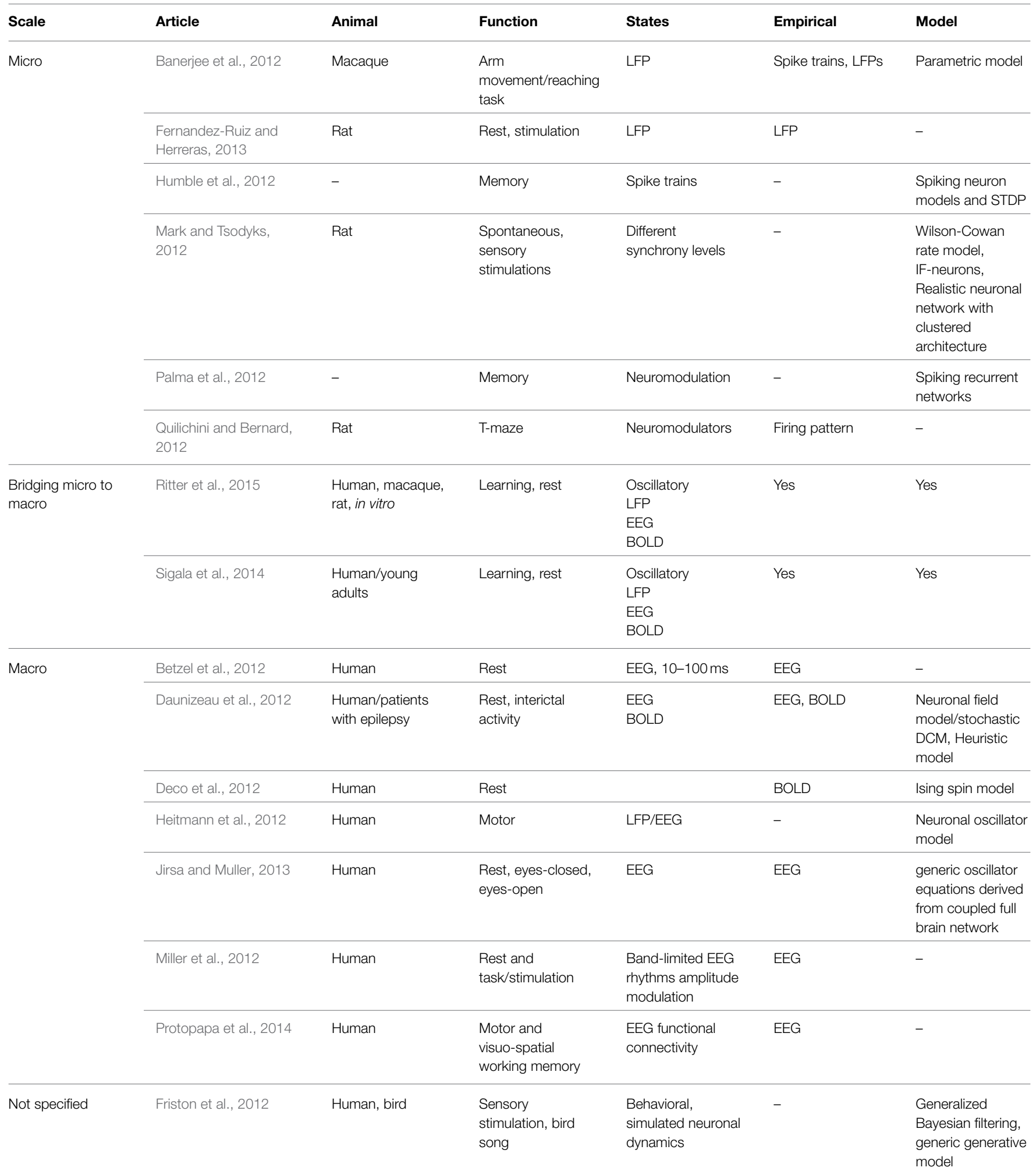

the human brain. Critical slowing caused by dynamical instabilities that are triggered by perception is proposed to enable the brain to process sensory perturbations (Friston et al.,
2012). Neuronal oscillator models with surround inhibition were shown to generate bistable spatial patterns of activity (Heitmann et al., 2012) and indicate that state-dependent computations 
may facilitate rapid switching between motor states, potentially accommodating high speed rather than precision responses. The cross-frequency coupling present in empirical EEG was systematically simulated in a full human brain network model of coupled neuronal oscillators (Jirsa and Muller, 2013) for eyesopen and eyes-closed states of rest condition and the theoretical implications for state-dependent processing discussed. Distinct brain states linked to motor and perceptual visuo-spatial working memory and accompanying specific mental processes are characterized as spatio-temporal functional connectivity patterns in EEG (Protopapa et al., 2014).

On the microscopic scale, spike trains and local field potentials (LFP) dynamics are set in relation (Banerjee et al., 2012) using parametric models with the goal to decode those signals and infer related behaviors. In Fernandez-Ruiz and Herreras (2013) it is pointed out that LFP's are highly variable over time and have flexible spectrums, i.e., the notion of periodic oscillations commonly used to describe brain activity is questioned. These authors propose a method to de-mix LFPs of different sources to determine the true degree of periodicity-a prerequisite for a mechanistic understanding of information transfer in the brain. In a spiking neuronal model with STDP (Humble et al., 2012) demonstrate that simple networks of laterally connected excitatory neurons can selforganize into spatio-temporal pattern recognizers. The potential for representations of more complex nested patterns which implies stronger computational memory capabilities is raised. The flow of information depends on the degree of network synchrony (Mark and Tsodyks, 2012) and an intermediate degree of synchrony is most beneficial for information transfer. The question whether rhythmic entrainment represents a general

\section{References}

Banerjee, A., Dean, H. L., and Pesaran, B. (2012). Parametric models to relate spike train and LFP dynamics with neural information processing. Front. Comput. Neurosci. 6:51. doi: 10.3389/fncom.2012.00051

Betzel, R. F., Erickson, M. A., Abell, M., O’Donnell, B. F., Hetrick, W. P., and Sporns, O. (2012). Synchronization dynamics and evidence for a repertoire of network states in resting EEG. Front. Comput. Neurosci. 6:74. doi: 10.3389/fncom.2012.00074

Daunizeau, J., Lemieux, L., Vaudano, A. E., Friston, K. J., and Stephan, K. E. (2012). An electrophysiological validation of stochastic DCM for fMRI. Front. Comput. Neurosci. 6:103. doi: 10.3389/fncom.2012.00103

Deco, G., Senden, M., and Jirsa, V. (2012). How anatomy shapes dynamics: a semianalytical study of the brain at rest by a simple spin model. Front. Comput. Neurosci. 6:68. doi: 10.3389/fncom.2012.00068

Fernandez-Ruiz, A., and Herreras, O. (2013). Identifying the synaptic origin of ongoing neuronal oscillations through spatial discrimination of electric fields. Front. Comput. Neurosci. 7:5. doi: 10.3389/fncom.2013.00005

Friston, K., Breakspear, M., and Deco, G. (2012). Perception and self-organized instability. Front. Comput. Neurosci. 6:44. doi: 10.3389/fncom.2012.00044

Heitmann, S., Gong, P., and Breakspear, M. (2012). A computational role for bistability and traveling waves in motor cortex. Front. Comput. Neurosci. 6:67. doi: 10.3389/fncom.2012.00067

Humble, J., Denham, S., and Wennekers, T. (2012). Spatio-temporal pattern recognizers using spiking neurons and spike-timing-dependent plasticity. Front. Comput. Neurosci. 6:84. doi: 10.3389/fncom.2012.00084

Jirsa, V., and Muller, V. (2013). Cross-frequency coupling in real and virtual brain networks. Front. Comput. Neurosci. 7:78. doi: 10.3389/fncom.2013.00078 mechanism of computation in the brain is raised and ways are pointed out how to address this question through empirical work in the future (Miller et al., 2012). The theoretical impact of neuromodulation on memory formation in spiking recurrent cortical networks is systematically evaluated (Palma et al., 2012). In a perspective article, a systematic account is provided how intrinsic properties of neurons and neuromodulation relates to firing patterns, functional correlations and behavior in rats (Quilichini and Bernard, 2012).

The degree of abstraction in the modeling work presented in this Research Topic varies tremendously, ranging from simplified but biophysically plausible network models to highly detailed neuron models. By placing the different mathematical and empirical aspects in this mutual context, this Research Topic aims to elucidate the principle mechanisms of statedependent neuronal processing. Developing a framework to link the multiple principles together is arguably the most pressing challenge. With The Virtual Brain (thevirtualbrain.org) simulation framework (Ritter et al., 2013; Sanz Leon et al., 2013) we hope to contribute to this endeavor by enabling researchers to use multiple modeling approaches in a unified framework ensuring reproducibility and comparability of results.

\section{Acknowledgments}

The authors acknowledge the support of the German Ministry of Education and Research (Bernstein Focus State Dependencies of Learning 01GQ0971) to PR, the James S. McDonnell Foundation (Brain Network Recovery Group JSMF22002082) to PR, VJ, $\mathrm{AM}, \mathrm{MB}$ and the Max-Planck Society (Minerva Program) to PR.

Mark, S., and Tsodyks, M. (2012). Population spikes in cortical networks during different functional states. Front. Comput. Neurosci. 6:43. doi: 10.3389/fncom.2012.00043

Miller, K. J., Foster, B. L., and Honey, C. J. (2012). Does rhythmic entrainment represent a generalized mechanism for organizing computation in the brain? Front. Comput. Neurosci. 6:85. doi: 10.3389/fncom.2012.00085

Palma, J., Grossberg, S., and Versace, M. (2012). Persistence and storage of activity patterns in spiking recurrent cortical networks: modulation of sigmoid signals by after-hyperpolarization currents and acetylcholine. Front. Comput. Neurosci. 6:42. doi: 10.3389/fncom.2012.00042

Protopapa, F., Siettos, C. I., Evdokimidis, I., and Smyrnis, N. (2014). Granger causality analysis reveals distinct spatio-temporal connectivity patterns in motor and perceptual visuo-spatial working memory. Front. Comput. Neurosci. 8:146. doi: 10.3389/fncom.2014.00146

Quilichini, P. P., and Bernard, C. (2012). Brain state-dependent neuronal computation. Front. Comput. Neurosci. 6:77. doi: 10.3389/fncom.2012.00077

Ritter, P., Born, J., Brecht, M., Dinse, H. R., Heinemann, U., Pleger, B., et al. (2015). State-dependencies of learning across brain scales. Front. Comput. Neurosci. 9:1. doi: 10.3389/fncom.2015.00001

Ritter, P., Schirner, M., McIntosh, A. R., and Jirsa, V. K. (2013). The virtual brain integrates computational modeling and multimodal neuroimaging. Brain Connect. 3:120. doi: 10.1089/brain.2012.0120

Sanz Leon, P., Knock, S. A., Woodman, M. M., Domide, L., Mersmann, J., McIntosh, A. R., et al. (2013). The Virtual Brain: a simulator of primate brain network dynamics. Front. Neuroinform. 7:10. doi: 10.3389/fninf.2013. 00010

Sigala, R., Haufe, S., Roy, D., Dinse, H. R., and Ritter, P. (2014). The role of alpha-rhythm states in perceptual learning: insights from 
experiments and computational models. Front. Comput. Neurosci. 8:36. doi: $10.3389 /$ fncom.2014.00036

Conflict of Interest Statement: The authors declare that the research was conducted in the absence of any commercial or financial relationships that could be construed as a potential conflict of interest.
Copyright $\odot 2015$ Ritter, Jirsa, McIntosh and Breakspear. This is an open-access article distributed under the terms of the Creative Commons Attribution License (CC $B Y$ ). The use, distribution or reproduction in other forums is permitted, provided the original author(s) or licensor are credited and that the original publication in this journal is cited, in accordance with accepted academic practice. No use, distribution or reproduction is permitted which does not comply with these terms. 